\title{
A successful staged approach for treatment of concomitant mitral insufficiency and mycotic aneurysm
}

\author{
Eric Zimmermann, MD, ${ }^{\mathrm{a}, \mathrm{b}}$ Takuya Ogami, MD, ${ }^{\mathrm{a}}$ Rajeev Dayal, MD, ${ }^{\mathrm{a}}$ and \\ Dimitrios V. Avgerinos, MD, PhD, ${ }^{\mathrm{c}}$ Flushing and New York, NY, and Portland, Ore
}

\footnotetext{
From the ${ }^{a}$ Department of Surgery, NewYork-Presbyterian/Queens, Flushing, NY; ${ }^{\mathrm{b}}$ Knight Cardiovascular Institute, Oregon Health Sciences University, Portland, Ore; and ${ }^{\mathrm{c}}$ Department of Cardiothoracic Surgery, Weill Cornell Medicine, NewYork Presbyterian, New York, NY.

Dr Eric Zimmermann is supported by the National Institutes of Health NRSA Post-Doctoral Scholar Grant T32HL094294.

Disclosures: Authors have nothing to disclose with regard to commercial support.

Read at the meeting of the European Society of CardioVascular and Endovascular Surgery, Groningen, The Netherlands, May 23-25, 2019.

This work was performed at NewYork-Presbyterian/Queens, Flushing, NY.

Received for publication Oct 28, 2019; revisions received Oct 28, 2019; accepted for publication Nov 29, 2019; available ahead of print Jan 10, 2020.

Address for reprints: Eric Zimmermann, MD, 56-45 Main St, WLL-300, Flushing, NY 11355 (E-mail: eric. zimmermann.md@gmail.com).

JTCVS Techniques 2020;1:6-8

2666-2507

Copyright @ 2019 The Author(s). Published by Elsevier Inc. on behalf of The American Association for Thoracic Surgery. Published by Elsevier Inc. This is an open access article under the CC BY-NC-ND license (http:// creativecommons.org/licenses/by-nc-nd/4.0/).

https://doi.org/10.1016/j.xjtc.2019.11.007
}

$\checkmark$ Video clip is available online.

A 78-year-old gentleman with diabetes and hypertension presented with a febrile illness and 10 days of shoulder pain. Electrocardiogram on admission revealed right heart strain, and computed tomography angiogram demonstrated a 5.0- $\times 4.2-\times 1.8-\mathrm{cm}$ pseudoaneurysm of the aorta distal to the left subclavian artery (Figure 1, $A$ ). Echocardiogram revealed mitral vegetations and severe regurgitation with a preserved left ventricular ejection fraction of $50 \%$. Blood cultures revealed Streptococcus gallolyticus subspecies. Magnetic resonance imaging of the brain and spine revealed punctate hemorrhage along the corona radiata in the brain and osteomyelitis of L4-L5, establishing the diagnosis of septic emboli from endocarditis and associated aortic mycotic aneurysm.

Broad-spectrum antibiotics were initiated and subsequently narrowed to ceftriaxone and gentamicin per blood culture sensitivities. Following 6 weeks of antibiotics and repeat negative blood cultures, the patient agreed to surgery to address the mycotic aneurysm. We felt he would not survive a combined mitral valve repair (MVR) and open surgical debridement with extra-anatomic bypass, so we staged the repair starting with stabilization of the aneurysmal defect with a thoracic endovascular aneurysm repair (TEVAR).

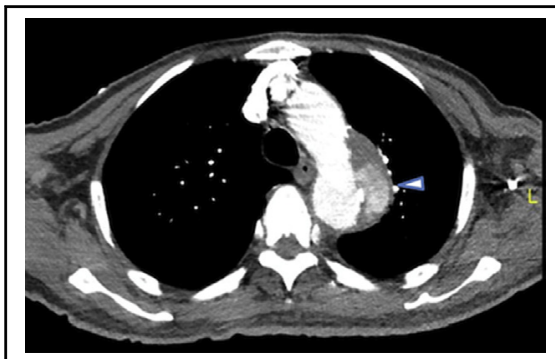

Staged repair of mycotic aneurysm (arrow) and mitral endocarditis.

CENTRAL MESSAGE

We report a case of staged endovascular and open

approach to treat concomitant

mitral endocarditis and aortic

mycotic aneurysm.

See Commentary on page 9.
Intraprocedural aortogram revealed a $3-\mathrm{cm}$ seal zone between the affected aorta and the left subclavian artery (Figure 1, B). A 32- × 109-mm Zenith Alpha thoracic endovascular graft (Cook Medical, Bloomington, Ind) was positioned and deployed with partial coverage of the left subclavian artery. Completion angiogram revealed exclusion of the pseudoaneurysm and preserved flow in the left subclavian artery (Figure 1, $C$ ). The patient tolerated the procedure well and was discharged on postoperative day 4 with lifelong suppressive oral antibiotics.

The patient was advised to undergo MVR after he recovered from the TEVAR but deferred surgery given his lack of symptoms and desire to avoid a large operation. Six months following discharge, the patient developed worsening shortness of breath related to mitral insufficiency. Echocardiogram demonstrated severe (4+) mitral regurgitation with associated flail A3/P3 segments, ruptured $\mathrm{A} 2 / \mathrm{P} 2$ chordae, $40-\mathrm{mm}^{2}$ regurgitant orifice, and regurgitant fraction of $62 \%(78 \mathrm{~mL})$. The patient now agreed to surgery, given his worsening symptoms. Intraoperatively, it was determined that the mitral valve was repairable via triangular plication of P3, commisuroplasty between A3 and P3, and annuloplasty with a 

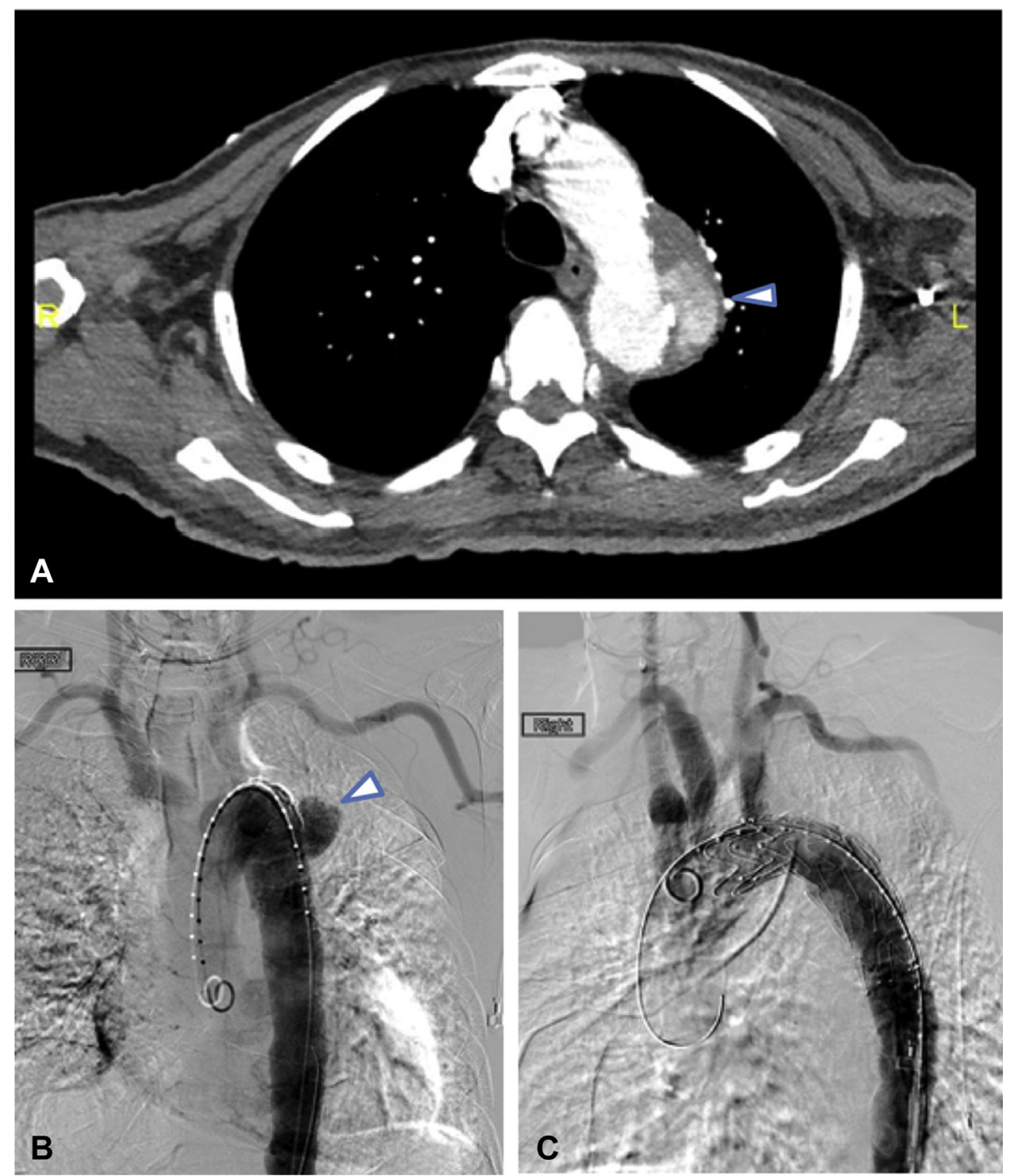

FIGURE 1. A, Computed tomography on admission shows contrast in a mycotic aneurysm defect (arrow). B, Location of defect with respect to left subclavian artery (arrow) before placement of stent graft. C, Completion angiogram demonstrates good left subclavian flow and exclusion of aortic defect

28-mm Physio II Edwards semirigid complete ring (Edward Lifesciences Corporation, Irvine, Calif). His clinical course was uncomplicated, and he was discharged to a skilled nursing facility on postoperative day 6 . At 24 months' follow-up, he has made a full recovery, maintains antibiotic compliance, and repeat blood cultures were negative; surveillance computed tomography angiogram shows no evidence of endoleak (Figure 2). The admission and follow-up imaging may be viewed in Video 1.

\section{DISCUSSION}

We chose to perform TEVAR before MVR because we felt stabilization of the aneurysm would mitigate risk of rupture in the eventual mitral intervention. There is agreement that all mycotic aneurysms rupture if left untreated; however, the risk of rupture at time of presentation ranges between $15 \%$ and $38 \%{ }^{1}$ It is up to the surgeon's clinical judgment to determine when to employ endovascular techniques for mycotic aneurysms due to concerns for recurrent graft infection and is ostensibly determined by the patient's tolerance for open surgical intervention. Immediate mortality appears to be similar between endovascular and open surgical repair $\left(5 \%\right.$ vs $4.8 \%$, respectively), ${ }^{2}$ and with adequate antimicrobial treatment before intervention, patients treated endovascularly have a greater survival than those treated with conventional surgery for abdominal mycotic aneurysms ( $80 \%$ vs $70 \%$ at 24 months, respectively). ${ }^{3}$

Collaborating with our infectious disease service, we treated the patient with antibiotics for 6 weeks and obtained 


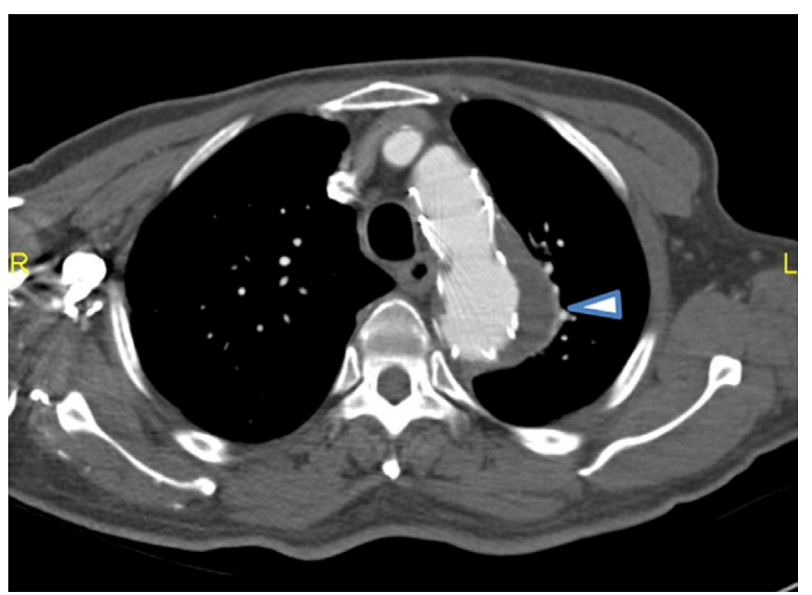

FIGURE 2. Follow-up computed tomography at 24 months with arrow pointing to residual aneurysm sack; there is no residual contrast filling the mycotic aneurysm.

negative blood cultures before TEVAR to protect against recurrent graft infection. Receiving antibiotics for greater than 1 week before intervention appears to be a protective factor. ${ }^{2}$ A separate nationwide study in Sweden reported a $71 \%$ five-year survival rate with median antibiotic treatment of 15 weeks before TEVAR. ${ }^{4}$ Anecdotal reports of soaking prosthetic grafts in rifampin before implantation reportedly help prevent secondary infection, but this practice remains controversial. ${ }^{1}$

If stigmata of infection such as leukocytosis or fever persist, F-fluorodeoxyglucose positron-emission tomography or radiolabeled white blood cell imaging may help localize ongoing infectious burden. ${ }^{5}$ If infective endocarditis or recurrent graft infection persists despite long-term antibiotic therapy, mitral valve replacement and excision of the graft with extra-anatomic bypass would be the only remaining option for long-term survival.

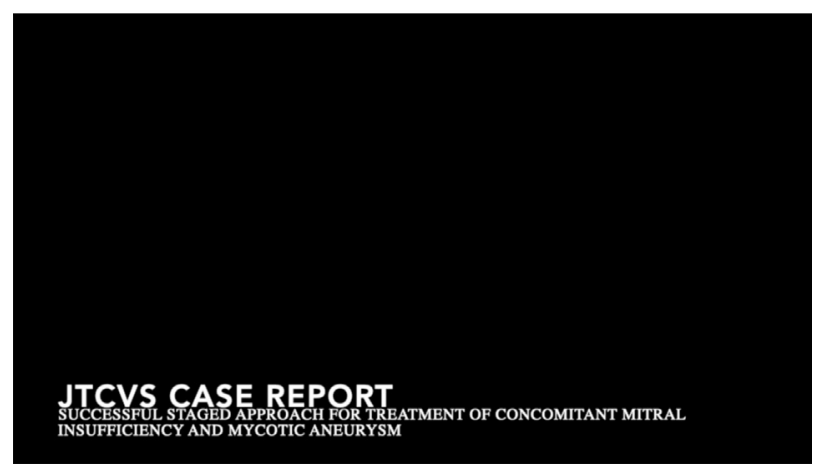

VIDEO 1. Video depicting initial computed tomography images, echo, and their respective follow-up studies. Video available at: https://www. jtcvs.org/article/S2666-2507(19)30057-4/fulltext.

The authors acknowledge the limitations of the current findings. Existing guidance relies primarily on retrospective reviews, which carry bias toward positive outcomes. To this end, prospective studies to establish higher-level evidence for treatment guidance may not be feasible, given the relatively rare nature of the disease.

\section{References}

1. Aoki C, Fukuda W, Kondo N, Minakawa M, Taniguchi S, Daitoku K, et al. Surgical management of mycotic aortic aneurysms. Ann Vasc Dis. 2017;10:29-35.

2. Kan CD, Lee HL, Luo CY, Yang YJ. The efficacy of aortic stent grafts in the management of mycotic abdominal aortic aneurysm - institute case management with systemic literature comparison. Ann Vasc Surg. 2010;24:433-40.

3. Setacci C, de Donato G, Setacci F. Endografts for the treatment of aortic infection. Semin Vasc Surg. 2011;24:242-9.

4. Sörelius K, Wanhainen A, Wahlgren CM, Langenskiöld M, Roos H, Resch T, et al. Nationwide study on treatment of mycotic thoracic aortic aneurysms. Eur J Vasc Endovasc Surg. 2019;57:239-46.

5. Glaudemans AW, Signore A. FDG-PET/CT in infections: the imaging method of choice? Eur J Nucl Med Mol Imaging. 2010;37:1986-91. 